\title{
3D Body Measurements Using Active Millimeter Wave Technology
}

\author{
M. F. KARIM, B. LUO, A. R. LEYMAN, I. R. KHAN, K. H. SEAH, L. C. ONG* \\ Institute for Infocomm Research $\left(I^{2} R\right)$, Singapore
}

\begin{abstract}
This paper presents the development of a millimeter wave system to conduct 3D body measurements of a person without the need for him to remove his clothes. This method uses the commercial vector network analyser to transmit and receive a wideband signal centred at $35 \mathrm{GHz}$. The millimeter wave signal illuminates the target, penetrates the clothing and reflects off the surface of the body. The frequency- and time-domain information are analysed and processed to obtain the 3D image.
\end{abstract}

Keywords: 3D body scanning, millimeter wave, imaging

\section{Introduction}

It is a common practice for apparel buyers to try many clothes before they select the most suitable clothes, particularly for ladies' clothings where there is a wider variety of fashionable designs and patterns. Different people have different physical builds and it is a waste of money, and resources to produce clothes for display in fashion retail shops which do not fit the potential buyers. Eventually, these unsold clothes have to be written off and disposed of. Instead of producing physical clothings, virtual clothings can be generated and tried on by potential buyers. The 3D virtual system would be able to provide advice and suggestions to the customers based on their body dimensions and preferences. Customers can then tailor-specify the patterns, colour and design of the clothings to fit their taste and personalities. The challenge, therefore, is to obtain the body measurements without removing the clothes. Currently, researchers are working on different types of active sensors systems for the human body measurement.

Active millimeter-wave (MMW) imaging technology operates as short-range radar (radio detection and ranging) systems with a wavelength from $10 \mathrm{~mm}$ to $1 \mathrm{~mm}$ (i.e. 30 to $300 \mathrm{GHz}$ ). MMW technology has been shown that it can penetrate though dielectric materials, such as plastic or cloth, and are strongly reflected by metallic materials. In particular, by applying active millimeter wave imaging technology on the human body, it is possible to perform a whole body scan while the person remains fully clothed. These characteristics are also favourable for security and surveillance applications, i.e. for the detection of weapons concealed under people's clothing and in baggage at airports [1-4].

A new application of millimeter-wave scanning/imaging is in the construction of 3D body measurements of a person. A MMW signal illuminates the human body, penetrates through the clothing and reflects off the surface of the body. The reflected signals are analysed through image processing software and the 3D image of the person is reconstructed.

\section{Millimeter wave scanning system}

The millimeter wave measurement system operates at $35 \mathrm{GHz}$. It consists of a horn lens antenna with the bandwidth from $33-37 \mathrm{GHz}$. This horn lens antenna has a gain of $35 \mathrm{dBi}$ and a beamwidth of $2.6^{\circ}$. It is mounted on a motor-controlled stage which scans and sweeps the human body as shown in Figure 1. This customized $x-z$ scanner can travel a total distance of $1.1 \mathrm{~m}$ by $2.1 \mathrm{~m}$ with a maximum speed of $1.5 \mathrm{~m} / \mathrm{s}$, acceleration of $10 \mathrm{~m} / \mathrm{s}^{2}$ and the repeatability of $50 \mu \mathrm{m}$. Resolution of this scanner is $1 \mathrm{~mm}$. The $35 \mathrm{GHz}$ signal is generated and captured with an Agilent E8364B vector signal analyzer (VNA). A time domain analysis software in the VNA displays the signal power in time against the distance using the inverse Fourier transformation. The depth of the human body parts can be obtained from the time domain measurements. The $x-z$ coordinates can be determined from the encoder of the motor controlled stage.

* ongmichael@i2r.a-star.edu.sg; +65-6408-2389; www.i2r.a-star.edu.sg 


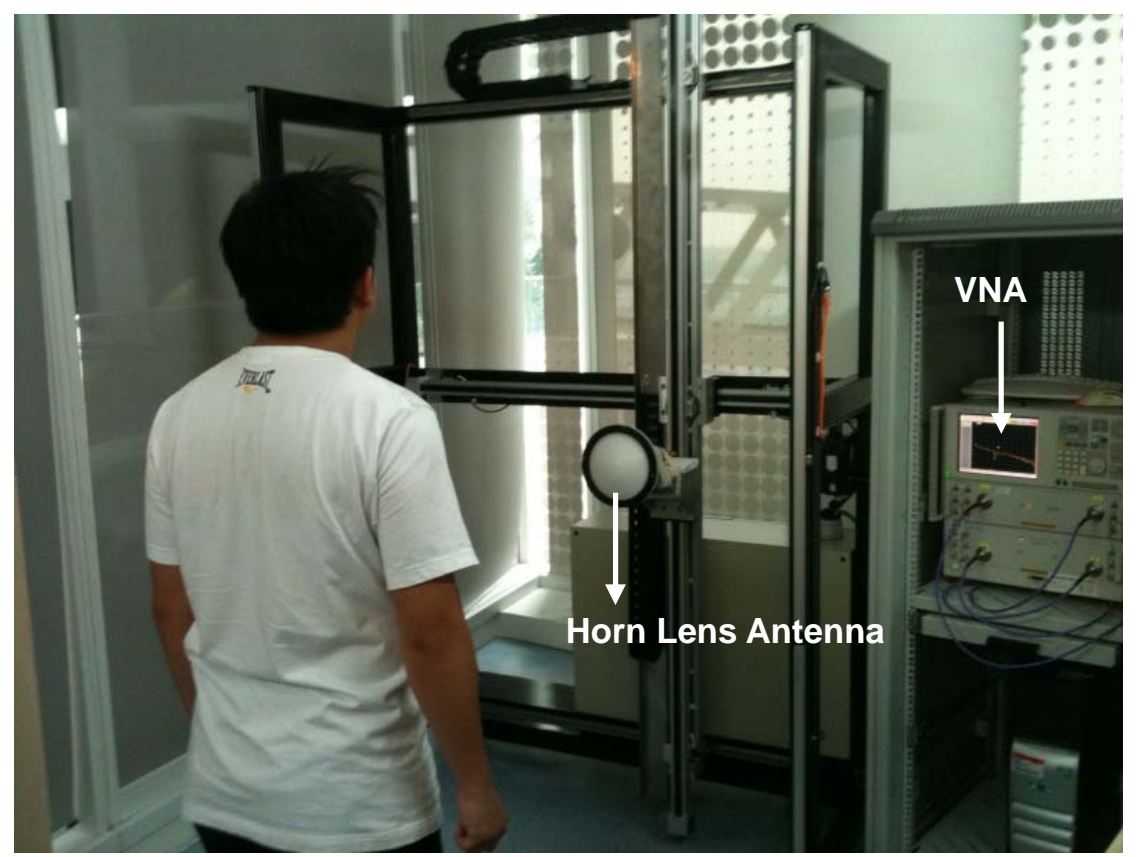

Fig. 1. Overview of the system setup at millimeter wave frequency.

Fig.2 illustrates the flow diagram of the MMW system controlled using the Labview software in the PC. This software is used to control the operations of the VNA and the scanner simultaneously. Both the frequency and time-domain information can be obtained using the VNA. Recently, it has been reported that it is possible to design an integrated, compact one-port vector reflectometer at our frequency of interest [5] and also a fast scanning system for advanced imaging applications [6].

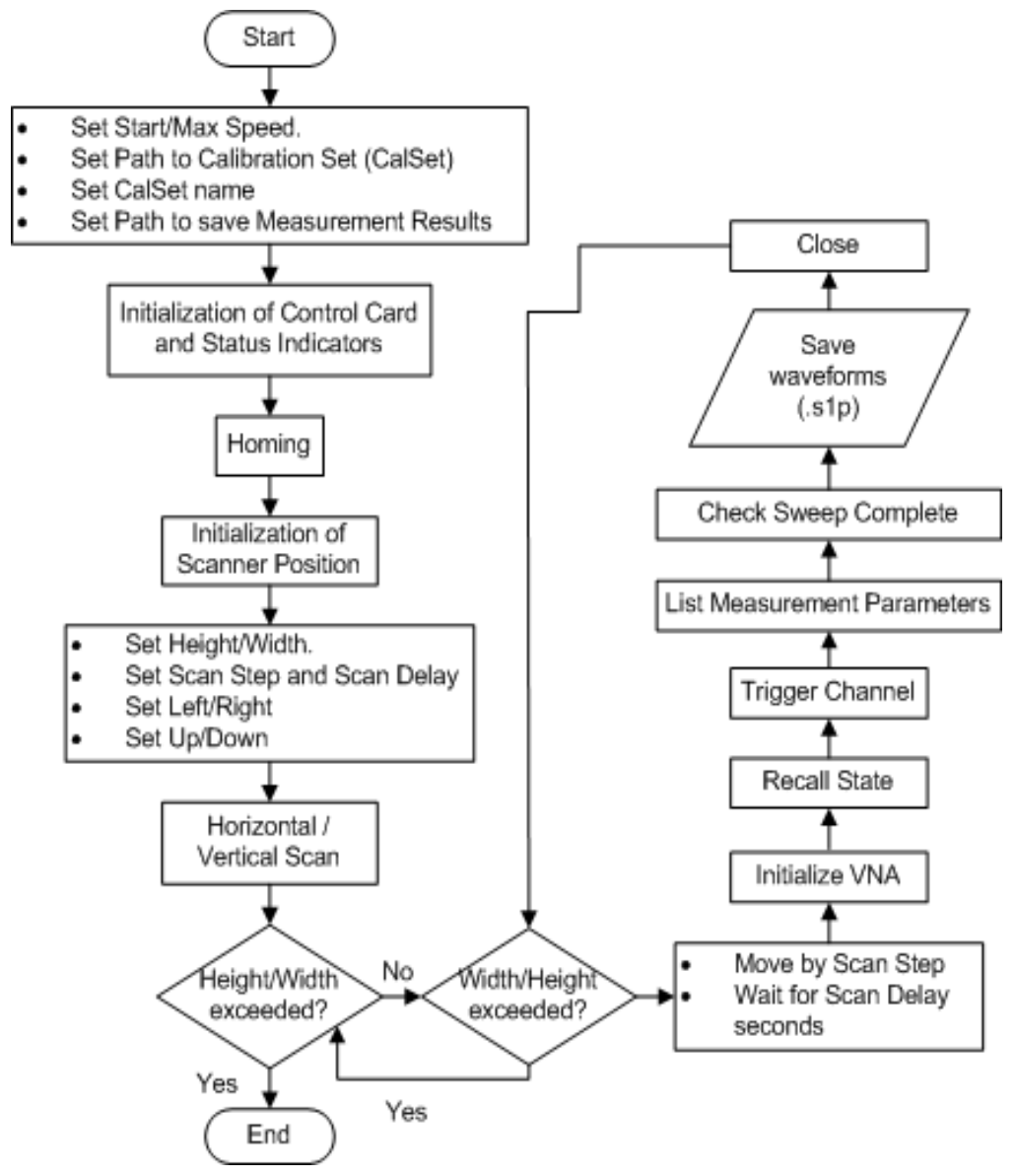

Fig. 2. Flow diagram of the MMW system. 


\section{Image processing}

One of the most practical problems in using millimeter wave technology is the requirement to restore the signal or image from a set of radar measurements as indicated in the previous section. Here, we consider the case where we do not have prior knowledge about the degradation phenomenon. We suspect that the image could have been heavily degraded by the sensing instruments (the millimeter acquisition system, noise and blocking artifacts) and the reflectivity environment. Given the frequency range we are operating in, mathematical modeling of this underlying degraded phenomenon is a cumbersome procedure. Hence, it is imperative that we focus on image restoration techniques that are primarily concerned with the reconstruction or recovery of the degraded image using well-known and tested two dimensional filters in both the spatial operations and frequency-transformed domains. We tried several linear and non-linear inversing techniques on the received 2-D data from the millimeter wave acquisition system. As we can observe, it is not always possible to obtain an accurate pseudo-inverse to mitigate these blurring effects.

In our application, the reflected signals are received by a single receiver. The image is reconstructed using these signals, with a sufficient amount of clutter presence. Although the grazing angle is fixed for each scan, the radar performance depends critically on its wavelength, surface roughness, and spatial characteristics of the said clutter.

\section{Results and discussions}

Millimeter wave scanning of the metal sphere, different shapes like star, octagon and triangle, and the mannequin are shown in Figures 3, 4 and 5. In the study of the metal ball, we are able to construct the image and a contour plots with received power.

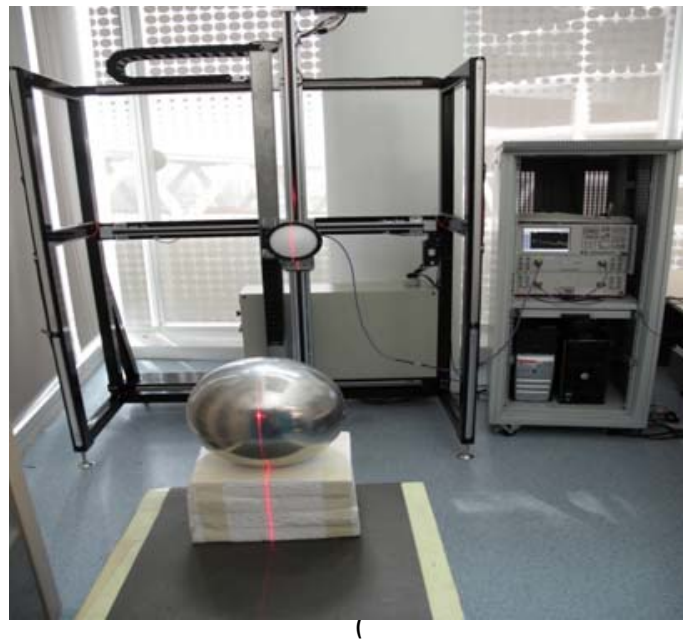

(a)

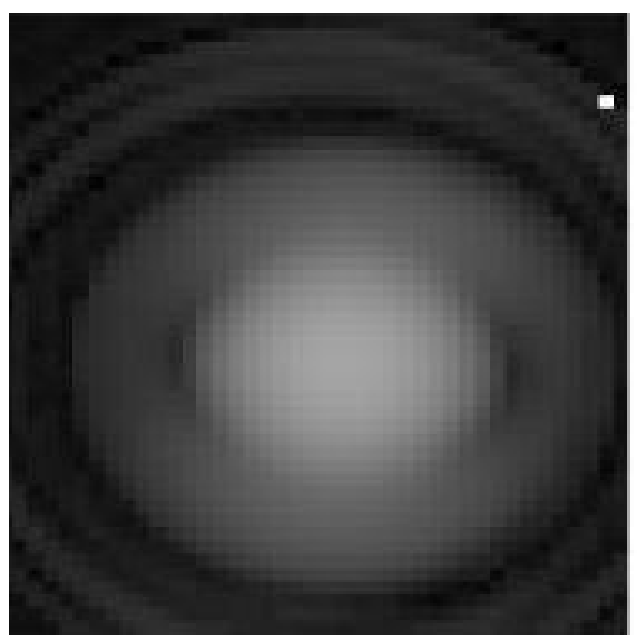

(b)

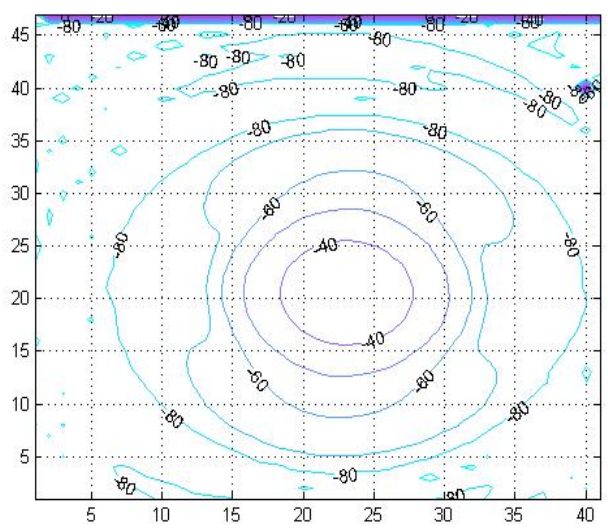

(c)

Fig. 3. Scanning of metal sphere, (a) photo of sphere; (b) millimeter wave image of sphere, and (c) sphere contour plot. 
We then studied the image formation of the three shapes as shown in Figure 4(a) and (b). The digital image was conveniently represented by an NXM matrix of amplitude signal strengths. These amplitudes define the physical properties of the scanned object, such as reflectivity, transmissivity and its multispectral response and the radar RCS. The matrix elements are integers in the range of [-70dB to $-10 \mathrm{~dB}]$. In the pre-processing stage, we smoothed the image over a convenient median filter to remove the "Salt and Pepper" noise found in front end retrieved data returns [7-8]. Next, we explicitly applied linear filters using several different masks to help in the restoration process. We experimented on the pass-band fluctuations and other transitions in the frequency domain. For example, shown in Figure 4(b) are the results obtained from the Prewitt and Log masks. It can be seen that the latter is more successful in providing better spatial resolution. In our point-operation experiments, we discovered that non-linear restoration and iterative techniques are not entirely useful in providing a good inverse.

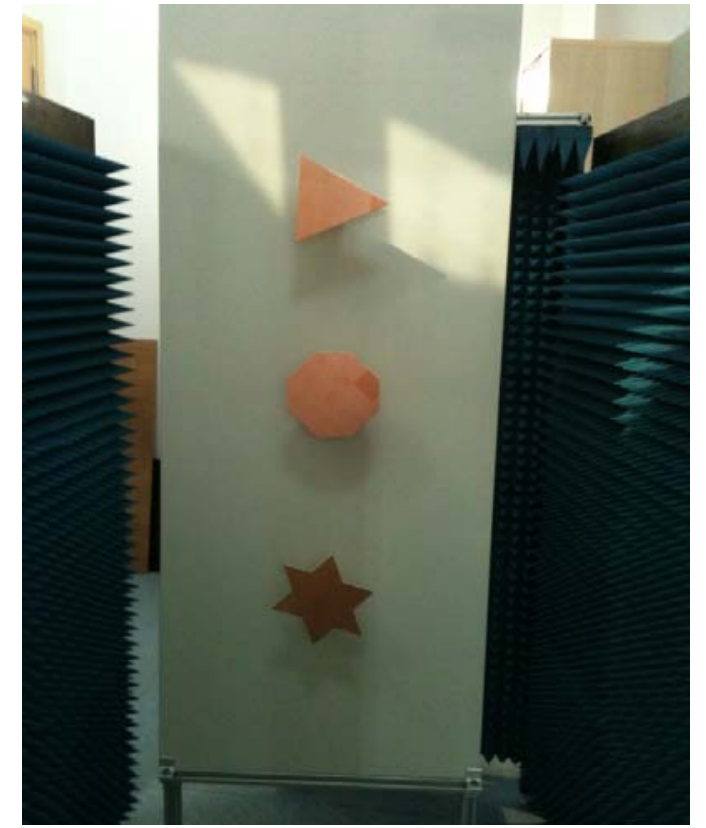

(a)

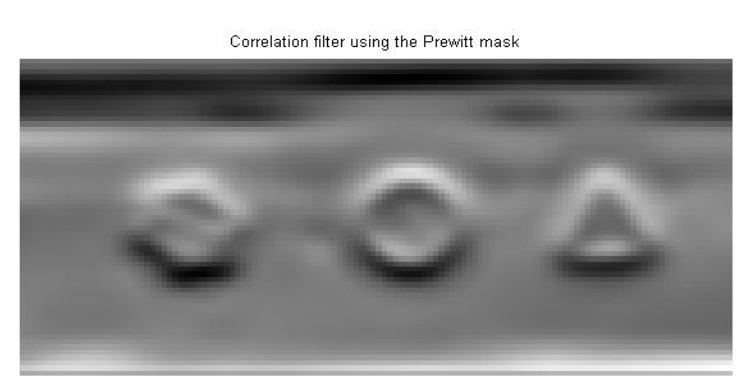

Correlation filter using the Log mask

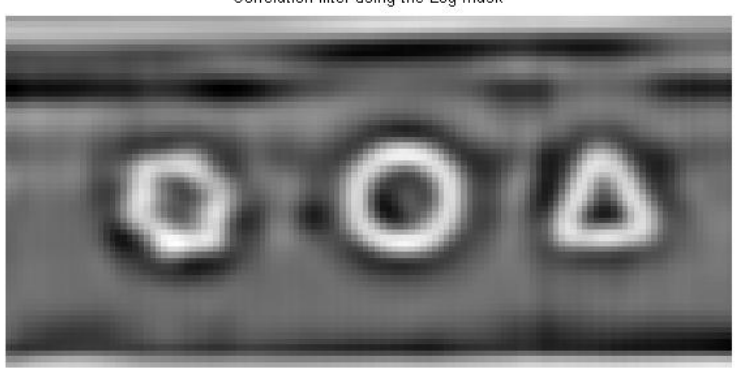

(b)

Fig. 4. Scanning of different shapes (a) photo and (b) millimeter wave images with different masks.

Next, we studied the image formation of a scanned mannequin shown in Figures 5 (a) and (b). Similarly, we implemented the above operations on the scanned mannequin data. Our difficulty lies in trying to reveal the mannequin details by simply applying the inverse linear and non-linear filtering techniques. Our experiments showed that the edges of the image are relatively low and the blurring level is strong, as observed in the connected boundaries. Small gaps can be seen from the filtered image, and edge linking was attempted using a $3 \times 3$ neighborhood to fill these gaps. We visually compared the image gradients of the performances of the several edge detection operators (Sobel, Prewitt, Roberts, Laplacian, zero-crossings and Canny). We concluded that the Canny method to be quite effective.

We carried out very extensive image restoration procedures to several different shapes and orientation, and studied the behavior of the millimeter wave returns under different degree of distinguishable details and different frequency bands. The perceived details are important in our application, and we seek to apply advance methods of image restoration, for example, the method of convex projections in our understanding of the distortive effects (amplitude and phase) of the parasitic returns that enter the radar through the antenna's main-lobe, and the nature of true image from millimeter wave returns. We need to further evaluate the performance of edge detection operators quantitatively on whether we would be able to precisely understand them in the context of millimeter wave returns. 


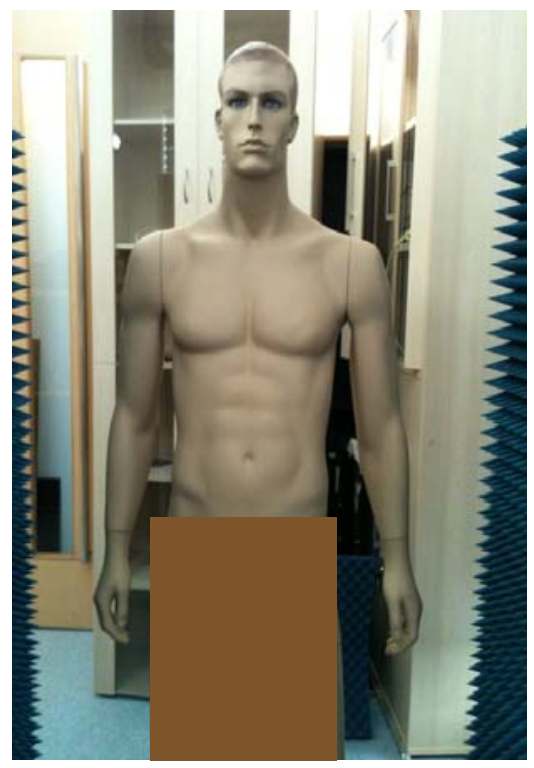

(a)

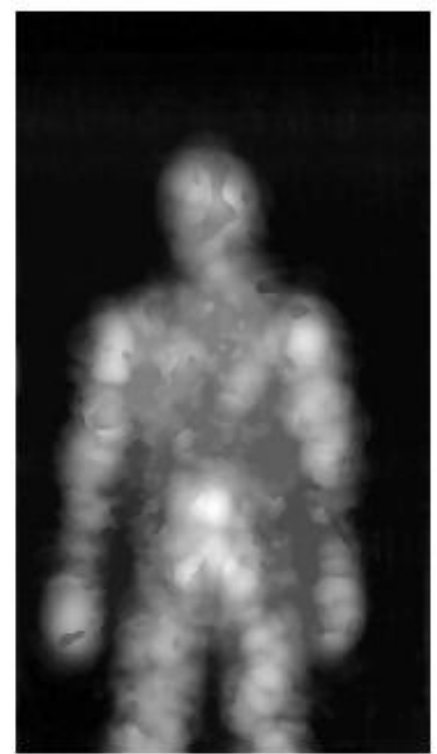

(b)

Fig. 5. Scanning of mannequin, (a) photo of mannequin and (b) millimeter wave image of mannequin.

\section{Conclusions}

We have developed a millimeter wave system at $35 \mathrm{GHz}$ and demonstrated its suitability for millimeter wave imaging applications. This system is able to penetrate clothing for 3D body measurements. The profiles of the scanned objects and mannequin can be constructed using the frequency domain information, while the time domain data provides the depth details. Good imaging techniques are required to reproduce these features with sufficient accuracy and resolution. This technique can also be used to reveal concealed objects hidden underneath the clothing for security and personnel screening.

\section{References}

1. Sheen, D. M., McMakin D. L. and Hall T. E. (2007): "Near field imaging at microwave and millimeter wave frequencies", Proc. of IEEE IMS 2007, Honolulu, USA, pp. 1693-1696.

2. Sheen, D. M., McMakin D. L. and Hall T. E. (2001): "Three-dimensional millimeter-wave imaging for concealed weapon detection", IEEE Trans. on Microwave Theory and Techniques, Vol. 49, No. 9, pp. 1581-1592.

3. Appleby, R and Anderton R. N. (2007): "Millimeter-wave and Submillimeter-wave Imaging for Security and Surveillance", Proc. Of IEEE, Vol. 95, No. 8, pp. 1683-1690.

4. Clark, S. E., Lovberg J. A., Martin C. A. and Kolinko V. (2003): "Passive millimeter-wave imaging for airborne and security applications", Proc. of SPIE, Vol. 5077, pp. 16-21.

5. Fallahpour, M., Baumgartner M., Ghasr M. T. and Zoughi R. (2011): "Intergated Design of a Compact Ka-band One-Port Vector Reflectormeter", Proc. Of IEEE I2MTC, Graz, Austria, pp. 1-5.

6. Tran, H. P., Gumbmann F., Weinzierl J. and Schmidt L. (2006): "A Fast Scanning W-band System for Advanced Millimetre-wave Short Range Imaging Applications", Proc. Of EuMA, Manchester, UK, pp. 146-149.

7. Lettington, A. H. and Hong Q.H. (1994): "Supperesolution Technique with Edge-based Ringing Reduction for Passive Millimetre-wave Images", IEE Proc. Vis. Image Signal Process, Vol. 141, No. 1, pp. 9-12.

8. Xu, Z. Y., Dou W. B. and Cao Z. X. (2008): "A New Algorithm for Millimeter-wave Imaging Processing", Proc. Joint China-Japan Microwave Conference, pp. 337-339. 